\title{
Defining an Intermediate-risk Group for Low-grade Glioma: A National Cancer Database Analysis
}

\author{
VIKRAM JAIRAM ${ }^{1}$, BENJAMIN H. KANN ${ }^{1}$, HENRY S. PARK ${ }^{1}$, JOSEPH A. MICCIO ${ }^{1}$, JASON M. BECKTA ${ }^{1}$, \\ JAMES B. YU ${ }^{1}$, ROSHAN S. PRABHU ${ }^{2}$, SARAH J. GAO ${ }^{1}$, MINESH P. MEHTA ${ }^{3}$, \\ WALTER J. CURRAN ${ }^{4}$, RANJIT S. BINDRA ${ }^{1}$, JOSEPH N. CONTESSA ${ }^{1}$ and KIRTESH R. PATEL ${ }^{1}$ \\ ${ }^{1}$ Department of Therapeutic Radiology, Yale School of Medicine, New Haven, CT, U.S.A.; \\ ${ }^{2}$ Southeast Radiation Oncology Group, Levine Cancer Institute, Charlotte, NC, U.S.A.; \\ ${ }^{3}$ Department of Radiation Oncology, Miami Cancer Institute, Baptist Health South Florida, Miami, FL, U.S.A.; \\ ${ }^{4}$ Department of Radiation Oncology, Winship Cancer Institute of Emory University, Atlanta, GA, U.S.A.
}

\begin{abstract}
Background: RTOG 9802 identified a cohort of patients with age less than 40 years and undergoing gross total resection as having low-risk, low-grade glioma (LR-LGG). European Organization for Research and Treatment of Cancer studies have demonstrated additional prognostic features in this group. The aim of this study was to analyze clinical factors associated with overall survival (OS), identify a potentially higher risk group within $L R-L G G$, and investigate patterns of care for adjuvant therapy. Materials and Methods: Patients with LR-LGG diagnosed between 2010 to 2013 were identified in the National Cancer Database. Kaplan-Meier method was used to analyze OS. Propensity score matching and multivariate analysis were utilized to adjust for differences in cohorts. Results: A total of 1,032 patients with $L R-L G G$ were identified. Histological breakdown was $42.0 \%$ astrocytoma, 33.2\% oligodendroglioma, and $25.8 \%$ mixed. Median follow-up was 3.9 years; median pre-operative tumor size was $4.0 \mathrm{~cm}$. Overall, 834 (80.8\%) underwent observation and 198 (19.2\%) received adjuvant therapy. Tumor size $>5 \mathrm{~cm}$ predicted for receipt of adjuvant therapy on regression analyses $(O R=2.02, p=0.001)$. On multivariate analysis, tumor size $>5 \mathrm{~cm}$ (hazard ratio=1.95) and non-oligodendroglioma histology (hazard ratio $=2.50$ ) were associated with inferior OS (both $p<0.05)$. For patients with both poor prognostic features (a subset we consider “intermediate-risk"), 5-year OS was $78.4 \%$, compared to
\end{abstract}

This article is freely accessible online.

Correspondence to: Vikram Jairam, MD, Department of Therapeutic Radiology, Yale University, 35 Park Street, LL509, New Haven, CT 06510, U.S.A. Tel: +1 2032002100, Fax: +1 2037854622, e-mail: vikram.jairam@yale.edu

Key Words: Low-grade glioma, low risk, stratification, overall survival, NCDB.
$94.1 \%$ for all other low-risk patients $(p<0.001)$. After propensity score matching, the intermediate-risk group continued to be associated with worse 5-year OS: $80.5 \%$ vs. 94.0\%, $p=0.004$. Conclusion: Due to inferior OS for patients with LR-LGG with $>5 \mathrm{~cm}$, non-oligodendroglioma tumors, we propose an 'intermediate-risk' clinical classification for this subset.

Low-grade gliomas (LGGs) are a heterogeneous and diffusely infiltrative group of neoplasms that comprise nearly $15 \%$ of all primary brain tumors (1). LGGs include World Health Organization (WHO) grade I and II tumors associated with median survival of approximately 13 years (2). The Radiation Therapy Oncology Group (RTOG) has classified grade 2 LGGs into low-risk (LR)-LGG based on two features: age $<40$ years, and undergoing gross total resection; patients not meeting both these criteria were termed as having high-risk (HR)-LGG (3). The RTOG 9802 study demonstrated that the addition of procarbazine, lomustine, and vincristine (PCV) to radiotherapy improved 5-year overall survival (OS) from $63 \%$ to $72 \%$ for those with HR-LGG. Meanwhile, for patients with LR-LGG undergoing observation, the 5-year OS was $94 \%$, while progression-free survival (PFS) was 50\% (4).

Similarly to the RTOG, The European Organization for Research and Treatment of Cancer (EORTC) also developed its own prognostic grouping for LGGs. Pignatti et al. pooled two clinical trials for LGG [EORTC 22844 Believers (5) and EORTC 22845 Non-Believers (6)] and identified five factors associated with OS: astrocytoma histology, age $\geq 40$ years, tumor $\geq 6 \mathrm{~cm}$, tumor crossing the midline, and neurologic functional deficit (7). Patients with LR-LGG as defined by RTOG may still carry one or more of these risk factors, which could portend a worse prognosis than that reported for the entire low-risk RTOG 9802 cohort (94\% 5-year OS).

With these variations in risk groups, practice patterns in LR-LGG may differ, with a number of these patients still receiving adjuvant therapy. The aim of this study was to use 
the National Cancer Database (NCDB) to investigate characteristics associated with receipt of adjuvant therapy and with OS for patients with LR-LGG. Additionally, this study attempted to identify a potentially higher risk group within those with LR-LGG.

\section{Materials and Methods}

Data source. The NCDB is a clinical oncology database maintained by both the American College of Surgeons and the American Cancer Society. Data are collected from hospital registries at more than 1,500 Commission on Cancer-accredited facilities. This database represents approximately $70 \%$ of newly diagnosed cancer cases in the United States. The NCDB contains detailed information on patient demographics, socioeconomic factors, tumor characteristics, and treatments delivered. All patient information in the NCDB is de-identified, and therefore this study was exempt from our university's Human Investigation Committee review.

Patient selection. The NCDB participant user file was queried for patients diagnosed with WHO grade II gliomas from 2010 to 2013. Extent of resection was not coded prior to 2010, therefore patients before this year were excluded. For our analysis, patients were only included if they had LGG meeting the RTOG 9802 definition of low-risk: adults age of 18 years to less than 40 years and undergoing gross total resection. Patients without histological confirmation were excluded. International Classification of Disease for Oncology [ICD-O-3] codes (8) included in our analysis were 9382, 9400, 9410, 9411, 9420, 9424, 9425, and 9450. Cases with missing outcomes were also excluded. We then defined two groups: Patients who underwent observation, and those who received adjuvant therapy, defined as chemotherapy or radiotherapy given within 180 days after surgery. Receipt of chemotherapy or radiation after 180 days was defined as salvage therapy and these patients were excluded from analysis. Figure 1 is a consort diagram showing the inclusion and exclusion criteria utilized in this study.

Variables. Demographic factors examined included year of diagnosis, age, sex, race, education, income, location (metropolitan vs. rural), and Charlson-Deyo score, a measure of comorbidities. A number of variables in the NCDB are only coded up to 2009, including facility location and facility type (academic vs. private), and could not be further assessed in our study. Disease characteristics included histology (mixed glioma, astrocytoma, or oligodendroglioma), tumor size ( $>5 v s . \leq 5 \mathrm{~cm})$, chromosome $1 \mathrm{p}$ loss of heterozygosity $(\mathrm{LOH})$, chromosome $19 \mathrm{q} \mathrm{LOH}$, midline extension, and focality (unifocal $v s$. multifocal). Follow-up time was defined as the interval between date of diagnosis and date of last contact or death. Adjuvant treatment characteristics included observation, chemotherapy alone, radiotherapy alone, or both chemotherapy and radiation.

Statistical analysis. Baseline differences between the observation and adjuvant therapy cohorts were assessed using chi-squared analysis for categorical covariates and analysis of variance for numerical covariates. The Wilcoxon rank-sum test was utilized to assess for differences in medians. Demographic and clinicopathologic predictors of observation $v s$. adjuvant therapy were analyzed using logistic regression analysis. Univariate and multivariate association between the study covariates and OS were estimated using Cox proportional hazards regression models. Multivariate modeling was carried out using a backwards stepwise selection method, removing all variables with a $p$-value $>0.20$.

OS was compared between risk groups using the Kaplan-Meier method and log-rank tests. Propensity score matching was utilized to reduce selection bias and balance demographic covariates between these groups. Matching was performed using clinically relevant demographic variables that were associated with the likelihood of being in a particular risk group including sex, race, income, education level, location, and Charlson-Deyo score. Analysis was performed with 1:1 nearest-neighbor matching without replacement. Covariate balance was evaluated between the matched cohorts using standardized difference of means. A value of less than 0.15 was considered a negligible imbalance. Survival analysis was then conducted. All statistical tests were two-sided and statistical significance was determined with $p \leq 0.05$ for all analyses. Data analysis was carried out using STATA v13.1 (StataCorp LP, College Station, TX, USA).

\section{Results}

Patient characteristics and predictors for receipt of adjuvant treatment. A total of 1,032 patients were included in our cohort: 834 (80.8\%) underwent observation and 198 (19.2\%) received adjuvant therapy. The median follow-up was 3.9 years. Within our cohort, $433(42.0 \%)$ had astrocytoma histology, 343 (33.2\%) had oligodendroglioma, and 256 (24.8\%) had mixed gliomas. Amongst patients receiving adjuvant therapy, $58(29.3 \%)$ received chemotherapy alone, 84 $(42.4 \%)$ received radiation alone, and $56(28.3 \%)$ received both chemotherapy and radiation. Amongst those who received both chemotherapy and radiation, 33 (58.9\%) received concurrent treatment, while $23(41.1 \%)$ received sequential therapy. Median tumor size was $4.0 \mathrm{~cm}$. On unadjusted univariate analysis, patients receiving adjuvant treatment were very slightly older (median $31 \mathrm{vs} .30$ years; $p=0.001$ ), more likely to have non-oligodendroglial histology (73.2\% vs. 65.2\%; $p=0.032)$, tumor size $>5 \mathrm{~cm},(32.3 \% v s$. $19.9 \% ; p=0.001)$, multifocal tumor $(6.4 \% v s .1 .5 \% ; p<0.001)$, midline extension $(5.6 \%$ vs. $2.7 \% ; p=0.036)$ and a longer median follow-up time (51.5 vs. 45.9 months; $p<0.001$ ). The median dose of radiotherapy delivered was $54.0 \mathrm{~Gy}$ and the median time to start of adjuvant treatment after surgery was 50 days (interquartile range $=29-83$ days) for patients overall. Amongst patients who received chemotherapy, $87.8 \%$ received single-agent chemotherapy, $4.3 \%$ received multiagent chemotherapy, and $7.9 \%$ had unknown chemotherapy types and agents. Table I lists baseline patient characteristics stratified by receipt of adjuvant treatment.

On multivariate logistic regression, only tumor size $>5 \mathrm{~cm}$ [odds ratio $(\mathrm{OR}) 2.02$; confidence intervaI $(\mathrm{CI})=1.34-3.04$; $p=0.001]$ was associated with increased odds of receiving adjuvant treatment. Histology and multi-focal tumor were not associated with receipt of adjuvant therapy. 


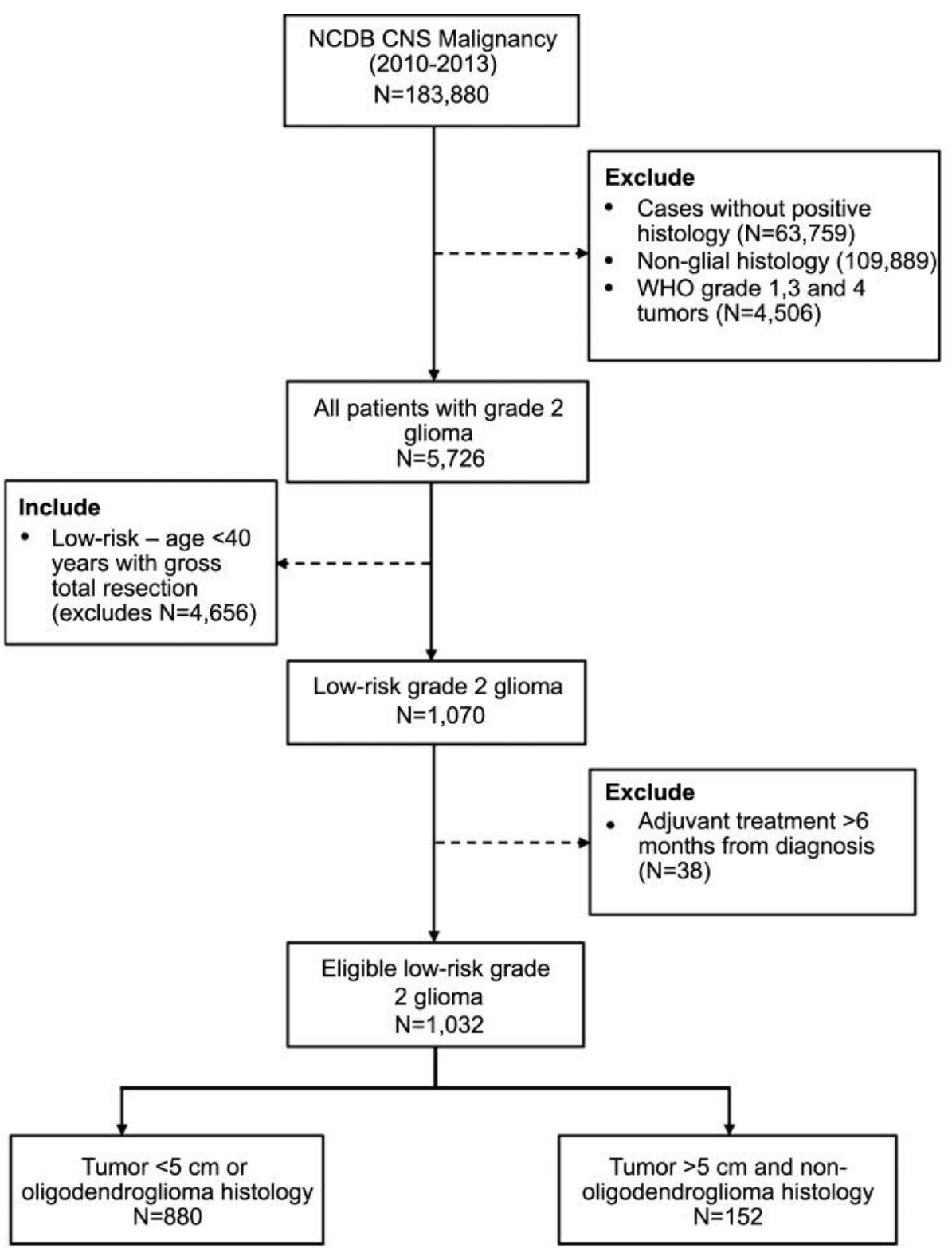

Figure 1. Consolidated Standards of Reporting Trials diagram for selection of patient cohort.

Overall survival. On univariate analysis for OS, income $<\$ 38,000$ per year (hazard ratio $(\mathrm{HR})=1.88,95 \% \mathrm{CI}=1.02$ $3.48 ; p=0.043$ ), non-oligodendroglial histology (HR 2.69; $\mathrm{CI}=1.45-5.00 ; p=0.002)$, and tumor size $>5 \mathrm{~cm}(\mathrm{HR}=2.27$; $\mathrm{CI}=1.22-4.22 ; p=0.01)$ were significantly associated with decreased survival. $1 \mathrm{p} / 19 \mathrm{q}$ co-deletion was not associated with OS $(p=0.372)$. Table II lists all covariates and their association with OS on univariate analysis. On multivariate analysis, size $>5 \mathrm{~cm}(\mathrm{HR}=1.95,95 \% \mathrm{CI}=1.08-3.50 ; p=0.03)$ and non-oligodendroglial histology $(\mathrm{HR}=2.50,95 \% \mathrm{CI}=1.21$ $5.18 ; p=0.02)$ remained significant for OS. Income was not associated with OS $(p=0.18)$. The 5-year OS for the entire cohort without any exclusions was $91.7 \%$.

Subgroup analysis. Patients with non-oligodendroglial histology and tumor size $>5 \mathrm{~cm}$ were grouped into an 'intermediate-risk' category. All others were categorized as 'low-risk'. There were 152 intermediate-risk patients, comprising $14.7 \%$ of the entire cohort. A total of $30.3 \%$ of intermediate-risk patients received adjuvant treatment compared to $19.2 \%$ of low risk patients $(p<0.001)$. Intermediate-risk patients were found to have significantly 
Table I. Baseline patient characteristics stratified by treatment type.

\begin{tabular}{|c|c|c|c|c|}
\hline \multirow[t]{2}{*}{ Variable } & & \multicolumn{2}{|c|}{ Cohort } & \multirow[t]{2}{*}{$p$-Value } \\
\hline & & Observation $(n=834)$ & Adjuvant $(\mathrm{n}=198)$ & \\
\hline \multirow[t]{2}{*}{ Age, years } & Mean & $29.53 \pm 6$ & $30.96 \pm 6$ & 0.001 \\
\hline & Median & 30 & 32 & \\
\hline \multirow[t]{2}{*}{ Gender, n (\%) } & Male & $446(53.48)$ & $98(49.49)$ & 0.313 \\
\hline & Female & $388(46.52)$ & $100(50.51)$ & \\
\hline \multirow[t]{2}{*}{ Race, $\mathrm{n}(\%)$} & White & $732(87.77)$ & $171(86.36)$ & 0.112 \\
\hline & Other & $89(10.67)$ & $27(13.64)$ & \\
\hline \multirow[t]{4}{*}{ No high school diploma, $\mathrm{n}(\%)^{*}$} & $\geq 29 \%$ & $127(15.53)$ & $21(10.71)$ & 0.094 \\
\hline & $20 \%-28.9 \%$ & $163(19.93)$ & $31(15.82)$ & \\
\hline & $14 \%-19.9 \%$ & $181(22.13)$ & $60(30.61)$ & \\
\hline & $<14 \%$ & $347(42.42)$ & $84(42.86)$ & \\
\hline \multirow[t]{4}{*}{ Median income quartile, $\mathrm{n}(\%)^{*}$} & $<\$ 38,000$ & $140(16.83)$ & $21(10.66)$ & 0.103 \\
\hline & $\$ 38,000-47,999$ & $171(20.55)$ & $37(18.78)$ & \\
\hline & $\$ 48,000-62,999$ & $212(25.48)$ & $61(30.96)$ & \\
\hline & $\geq \$ 63,000$ & $309(37.14)$ & $78(39.59)$ & \\
\hline \multirow[t]{2}{*}{ Geographic location, n (\%) } & Metropolitan & $681(84.07)$ & $163(85.34)$ & 0.665 \\
\hline & Urban/rural & $129(15.93)$ & $28(14.66)$ & \\
\hline \multirow[t]{2}{*}{ Charlson-Deyo score, n (\%) } & 0 & $740(89.16)$ & $178(89.90)$ & 0.761 \\
\hline & $\geq 1$ & $90(10.84)$ & $20(10.10)$ & \\
\hline \multirow[t]{4}{*}{ Year of diagnosis, n (\%) } & 2010 & $205(24.58)$ & $67(33.84)$ & 0.056 \\
\hline & 2011 & $195(23.38)$ & $42(21.21)$ & \\
\hline & 2012 & $213(25.54)$ & $40(20.20)$ & \\
\hline & 2013 & $221(26.50)$ & $49(24.75)$ & \\
\hline \multirow[t]{3}{*}{ Histology, n (\%) } & Mixed glioma & $189(22.66)$ & $67(33.84)$ & 0.003 \\
\hline & Astrocytoma & $355(42.57)$ & $78(39.39)$ & \\
\hline & Oligodendroglioma & $290(34.77)$ & $53(26.77)$ & \\
\hline \multirow[t]{5}{*}{ Tumor size, n (\%) } & $\leq 5 \mathrm{~cm}$ & $459(55.04)$ & $89(44.95)$ & 0.001 \\
\hline & $>5 \mathrm{~cm}$ & $166(19.90)$ & $64(32.32)$ & \\
\hline & Unknown & $209(25.06)$ & $45(22.73)$ & \\
\hline & Mean \pm SD, mm & $39.95 \pm 18$ & $48.82 \pm 19$ & $<0.001$ \\
\hline & Median, mm & 37 & 47 & \\
\hline \multirow[t]{4}{*}{ Codeletion of 1p19q, n (\%) } & Both positive & $103(12.35)$ & $24(12.12)$ & 0.966 \\
\hline & Either negative & $22(2.64)$ & $5(2.53)$ & \\
\hline & Both negative & $147(17.63)$ & $38(19.19)$ & \\
\hline & Unknown & $562(67.63)$ & $131(66.16)$ & \\
\hline \multirow[t]{3}{*}{ Tumor number, n (\%) } & Unifocal & $730(87.53)$ & $166(83.84)$ & 0.001 \\
\hline & Multifocal & $12(1.44)$ & $12(6.06)$ & \\
\hline & Unknown & $92(10.3)$ & $20(10.10)$ & \\
\hline \multirow[t]{2}{*}{ Tumor extension, n (\%) } & Crossing midline & $22(2.67)$ & $11(5.61)$ & 0.036 \\
\hline & Not crossing midline & $803(97.33)$ & $185(94.39)$ & \\
\hline \multirow[t]{2}{*}{ Time to adjuvant therapy, n (\%) } & $\leq 3$ Months & - & $154(77.78)$ & - \\
\hline & $>3$ Months & - & $44(22.22)$ & \\
\hline \multirow[t]{2}{*}{ Follow-up time, months } & Mean \pm SD & $45.36 \pm 19$ & $51.02 \pm 18$ & $<0.001$ \\
\hline & Median & 45.95 & 51.53 & \\
\hline
\end{tabular}

*Chi-squared tests compared $\geq 13 \%$ vs. $<13 \%$ for proportion without high school diploma in patient's area of residence; $\geq \$ 63 \mathrm{k} v s .<\$ 63 \mathrm{k}$ for income. SD: Standard deviation.

worse OS compared to low risk (HR 3.04, 95\% CI=1.87$4.95 ; p<0.001)$. Median survival was not reached in either group. Two-year OS was similar for the intermediate-risk and low-risk groups $(96.5 \%$ vs. 98.2\%; log-rank $p<0.704)$. This became a statistically significant survival difference with longer follow-up at 5 years $(78.4 \% v s .94 .1 \%$; log-rank $p<0.001)$. Amongst intermediate-risk patients, 5-year OS was not significantly different between those who underwent observation and those who received adjuvant treatment (76.8\% vs. 81.8\%; log-rank $p=0.617$ ).

Baseline patient characteristics and demographic variables were then balanced using 1:1 propensity score matching. Table III lists the balanced covariates with standardized difference $<0.15$. A total of 296 patients were matched in our 
Table II. Univariate analysis for overall survival.

\begin{tabular}{|c|c|c|c|c|}
\hline \multirow[b]{2}{*}{ Covariate } & \multirow[b]{2}{*}{ Level } & \multirow[b]{2}{*}{ HR $(95 \% \mathrm{CI})$} & \multicolumn{2}{|c|}{$p$-Value } \\
\hline & & & HR & Log-rank \\
\hline \multirow[t]{2}{*}{ Cohort } & Adjuvant treatment & $1.47(0.89-2.44)$ & 0.136 & 0.134 \\
\hline & Observation & Ref & - & \\
\hline Year of diagnosis & Continuous & $0.93(0.72-1.20)$ & 0.595 & - \\
\hline Age, years & Continuous & $0.97(0.94-1.02)$ & 0.219 & - \\
\hline \multirow[t]{2}{*}{ Gender } & Male & $1.42(0.88-2.28)$ & 0.150 & 0.148 \\
\hline & Female & Ref & - & \\
\hline \multirow[t]{2}{*}{ Race } & White & $1.07(0.51-2.24)$ & 0.855 & 0.855 \\
\hline & Other & Ref & - & \\
\hline \multirow[t]{2}{*}{ No high school education } & $\geq 13 \%$ & $1.53(0.95-2.45)$ & 0.080 & 0.078 \\
\hline & $<13 \%$ & Ref & - & \\
\hline \multirow[t]{2}{*}{ Median income } & $\geq \$ 63,000$ & $0.78(0.48-1.28)$ & 0.321 & 0.31 \\
\hline & $<\$ 63,000$ & Ref & - & \\
\hline \multirow[t]{2}{*}{ Charlson-Deyo score } & $1+$ & $1.40(0.72-2.74)$ & 0.321 & 0.319 \\
\hline & 0 & Ref & - & \\
\hline \multirow[t]{2}{*}{ Geographic location } & Metro & $0.85(0.46-1.59)$ & 0.612 & 0.612 \\
\hline & Urban/rural & Ref & - & \\
\hline \multirow[t]{3}{*}{ Histology } & Mixed glioma & $2.87(1.44-5.71)-$ & 0.003 & 0.005 \\
\hline & Astrocytoma & $2.57(1.33-4.96)$ & 0.005 & \\
\hline & Oligodendroglioma & Ref & - & \\
\hline \multirow[t]{3}{*}{ Tumor size } & $\leq 5 \mathrm{~cm}$ & $0.90(0.48-1.69)$ & 0.735 & $<0.001$ \\
\hline & $>5 \mathrm{~cm}$ & $2.27(1.22-4.22)$ & 0.010 & \\
\hline & Unknown & Ref & - & \\
\hline \multirow[t]{4}{*}{ Co-deletion of $1 \mathrm{p} 19 \mathrm{q}$} & Both positive & $0.45(0.16-1.24)$ & 0.121 & 0.372 \\
\hline & Either negative & $1.07(0.26-4.39)$ & 0.928 & \\
\hline & Both negative & $1.17(0.66-2.09)$ & 0.591 & \\
\hline & Unknown & Ref & - & \\
\hline \multirow[t]{3}{*}{ Tumor number } & Unifocal & $1.13(0.41-3.10)$ & 0.820 & 0.311 \\
\hline & Multifocal & $2.66(0.59-11.87)$ & 0.201 & \\
\hline & Other/unknown & Ref & - & \\
\hline \multirow[t]{2}{*}{ Tumor extension } & Crossing midline & $1.27(0.40-4.04)$ & 0.686 & 0.685 \\
\hline & Not crossing midline & Ref & - & \\
\hline \multirow[t]{2}{*}{ Time to adjuvant treatment } & $\leq 3$ Months & $0.91(0.30-2.70)$ & 0.859 & 0.859 \\
\hline & $>3$ Months & Ref & - & \\
\hline
\end{tabular}

cohort, with 148 each from the low-risk and intermediaterisk groups. Figure $2 \mathrm{~A}$ illustrates $\mathrm{OS}$ by risk group for the entire cohort, while Figure 2B depicts OS of the propensity score-matched group. At 5 years, OS for the low-risk (94.0\%; 95\% CI=87.5-97.1\%) and intermediate-risk (80.5\%; $95 \% \mathrm{CI}=70.4 \%-87.4 \%$ ) groups remained statistically different (log-rank $p=0.004)$.

\section{Discussion}

Our analysis suggests that for low-risk LGG, clinical factors exist that predict for inferior OS, including nonoligodendroglial histology and tumor size $>5 \mathrm{~cm}$; patients with both of these characteristics were deemed at 'intermediate-risk'. Five-year OS was lower for the intermediate-risk group: $78.4 \%$ vs. $94.1 \%$ (log-rank $p<0.001$ ), which held up on propensity-score matching. Finally, these data demonstrated that only tumor size $>5 \mathrm{~cm}$ was the primary factor which predicted for receipt of adjuvant treatment amongst patients with LR-LGG.

The identification of two prognostic factors for OS, namely tumor size $>5 \mathrm{~cm}$ and non-oligodendroglial histology, are consistent with previously reported studies. Pignatti et. al. reported on prognostic factors of OS in adult patients with LGG and found that tumor size $\geq 6 \mathrm{~cm}$ and astrocytoma histology both predicted for reduced OS (7). The other prognostic factor of Pignatti et al. explored in our analysis was tumor crossing midline, which was not significant on UVA or MVA. However, while tumor size and histology have been shown to be strong risk factors in LGG in multiple randomized trials $(5,6,9)$ tumor crossing the midline is less consistently found to be a risk factor (10). 
A

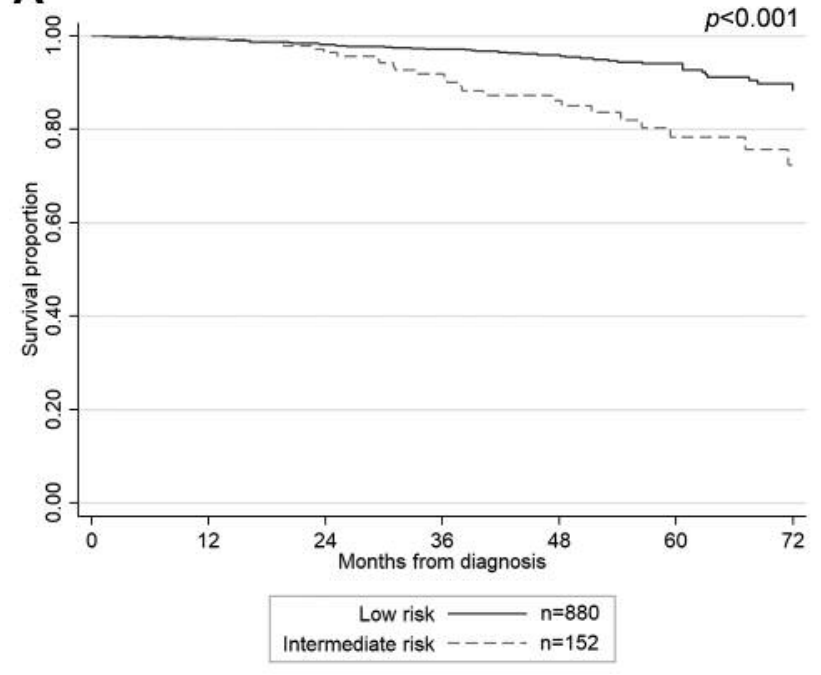

B

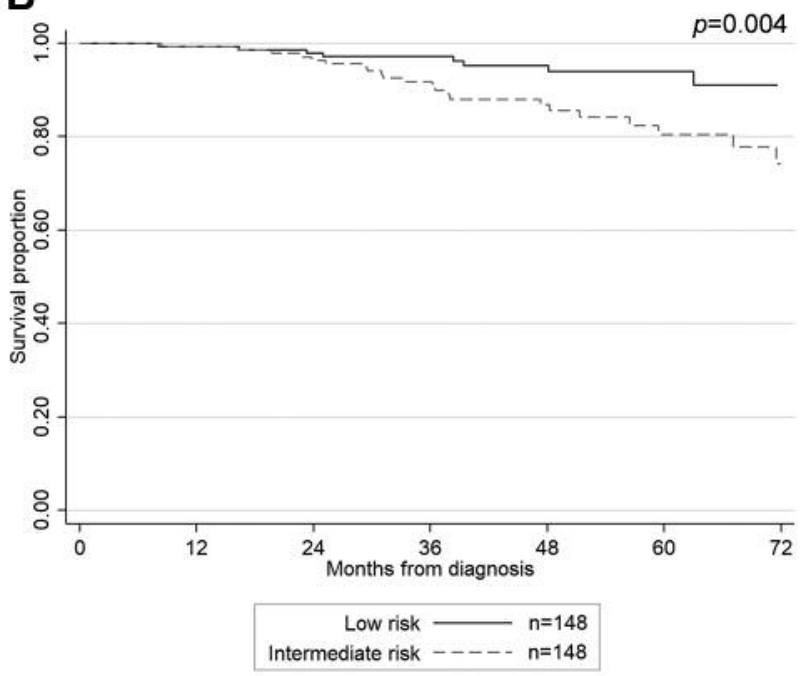

Figure 2. Kaplan-Meier survival analysis of the entire cohort (A) and propensity-score-matched patients (B) stratified by risk group.

Much of this analysis was also carried out in an era before molecular genetics were used to stratify patients with LGG. Recent genomic studies have shown that isocitrate dehydrogenase $(I D H)$ mutation, $O^{6}$-methylguanine-DNA methyltransferase $(M G M T)$ gene promoter methylation, and $1 \mathrm{p} 19 \mathrm{q}$ co-deletion are prognostic of improved OS or response to chemotherapy in LGG (11-13). Indeed, the recent 2016 WHO CNS classification update includes molecular markers to better define CNS tumors (14). For example, the diagnosis of oligodendroglioma requires the presence of an $I D H$ gene mutation and co-deletion of 1p19q. In fact, in the case of discordance between genetics and pathology for diffuse gliomas, the genetic features trump the histologicaI phenotype. Although our study did not show any survival difference associated with $1 \mathrm{p} 19 \mathrm{q}$ co-deletion, this information was unknown in approximately two-thirds of our cohort (Table I), precluding any meaningful conclusions from being drawn.

RTOG 9802 demonstrated 5-year OS of $93 \%$ for the LRLGG group (3). Consistent with these findings, we also report a similar 5-year OS of $91.7 \%$ for our entire cohort and $94.0 \%$ for the propensity score-matched low-risk group. In contrast, the 'intermediate-risk' group as defined by us had worse 5-year OS: $78.4 \%$ overall and $80.5 \%$ after propensity score matching. These results suggest that based on available clinical criteria, the intermediate-risk group can be identified as a possible subset of patients with LR-LGG at risk of worse OS. Validation of these findings from prospective clinical trials are required to support risk stratification into an intermediate-risk group.

It was also notable that a higher percentage of intermediate-risk patients received adjuvant treatment $(30 \%$
Table III. Propensity score matching showing standardized differences $<0.15$ between intermediate-risk and low-risk groups.

\begin{tabular}{lccc}
\hline Variable & \multicolumn{2}{c}{ Cohort, n $(\%)$} & $\begin{array}{c}\text { Standardized } \\
\text { difference }\end{array}$ \\
\cline { 2 - 3 } & $\begin{array}{c}\text { Intermediate-risk } \\
(\mathrm{n}=152)\end{array}$ & $\begin{array}{c}\text { Low-risk } \\
(\mathrm{n}=880)\end{array}$ & \\
\hline Gender & & & \\
$\quad$ Male & $83(54.7)$ & $462(52.5)$ & 0.044 \\
$\quad$ Female & $69(45.3)$ & $418(47.5)$ & 0.044 \\
Race & & & \\
$\quad$ White & $135(88.6)$ & $784(89.1)$ & 0.015 \\
$\quad$ Non-White & $17(11.4)$ & $96(10.9)$ & 0.015 \\
Income & $59(38.7)$ & $324(36.8)$ & 0.038 \\
$\quad<\$ 63,000$ & $93(61.3)$ & $556(63.2)$ & 0.038 \\
$\quad \$ \$ 63,000$ & & & \\
High school education & $47(30.9)$ & $267(30.3)$ & 0.013 \\
$\quad \geq 13 \%$ & $105(69.1)$ & $613(69.7)$ & 0.013 \\
$\quad<13 \%$ & & & \\
Charlson-Deyo score & $130(85.3)$ & $791(89.9)$ & 0.140 \\
$\quad 0$ & $22(14.7)$ & $89(10.1)$ & 0.140 \\
$\quad \geq 1$ & $125(82.0)$ & $746(84.8)$ & 0.076 \\
Location & $27(18.0)$ & $134(15.2)$ & 0.076 \\
$\quad \begin{array}{l}\text { Metropolitan } \\
\text { Urban/rural }\end{array}$ & & & \\
\hline
\end{tabular}

vs. 20\%). Given that tumor size and histology are known risk factors, it is plausible that clinicians would be more likely to prescribe adjuvant treatment. Indeed, a phase II observational study of the low-risk cohort of RTOG 9802 found that preoperative tumor size $\geq 4 \mathrm{~cm}$ and astrocytoma or oligoastrocytoma histology predicted for increased recurrence 
(15). Although we did not find a survival difference with adjuvant therapy amongst the intermediate-risk group, this was overall a small subset of patients. With limited numbers, inherent baseline differences and large selection biases between adjuvant and observation groups come into play. Therefore, the true impact of adjuvant therapy cannot be determined. Furthermore, our median follow-up of 3.9 years also limits our ability to study the benefit of OS with adjuvant therapy, as prospective studies have only demonstrated an OS difference after 4 years (3).

Our study has several limitations. Firstly, the NCDB does not capture data on tumor recurrence, so we were unable to assess PFS amongst our cohort. Given that likely over $50 \%$ of our cohort experienced disease progression during the timeframe of our study, PFS would have enabled us to evaluate if the intermediate-risk group was at higher risk of progression and if their disease progressed sooner. In addition, there is an inherent selection bias regarding treatment allocation. We could not account for unmeasured cofounders such as patient functional status, facility volume, clinician expertise, or availability of technology. Thirdly, because gross total resection was only included as a variable beginning in 2010 , our study was limited to 3 years of data. This affected the median follow-up time of 3.9 years, which is short for a disease with survival extending decades, particularly for patients with low-risk disease. There were also demographic variables coded before 2010 that could not be assessed in this study, including facility type and facility location. Finally, our cohort had multiple covariates with unknown results including tumor size (25\% unknown) and $1 \mathrm{p} 19 \mathrm{q}$ status (67\% unknown), which may have contributed to selection bias in this study. The NCDB also lacked molecular data on IDH status and had limited information on MGMT methylation. In fact, we acknowledge that it is speculatively possible that knowledge of molecular variables could strongly complement or supplant such a clinical classification in LR-LGG. However, until we can demonstrate that to indeed be the case, we cannot ignore the results derived from clinical prognostic variables.

In conclusion, patients with tumor size $>5 \mathrm{~cm}$ and nonoligodendroglial histology comprised $15 \%$ of patients with LR-LGG. These patients had a significantly inferior OS, supporting risk stratification further into those with intermediate-risk. Prospective randomized trials incorporating molecular markers and investigating optimal adjuvant therapy for this class are warranted.

\section{Conflicts of Interest}

HSP reports receiving honoraria from Varian Medical Systems Inc and RadOncQuestions LLC. JBY reports receiving research funding from 21st Century Oncology and serving as a consultant for Augmentix. MPM reports serving as a consultant for Varian,
Oncoceutics, Astra-Zeneca, Celgene, Tocagen, Abbvie, on the Data Safety Monitoring Board for Monteris, and on the Board of Directors of Oncoceutics. RSB is the co-founder and paid consultant for Cybrexa Therapeutics, LLC and also the founder of Athena Therapeutics, LLC.

\section{Authors' Contributions}

Vikram Jairam: Conceptualization, methodology, visualization, writing-original draft, project administration, and writing-review and editing. Benjamin Kann: Writing-review and editing, formal analysis, data curation; Henry S. Park: Writing-review and editing, formal analysis, data curation; Joseph A. Miccio: Writing-review and editing; Jason M. Beckta: Writing-review and editing; James B. Yu: Writing-review and editing, software; Roshan S. Prabhu: Writing-review and editing; Sarah J. Gao: Writing-review and editing; Minesh P. Mehta: Writing-review and editing; Walter J. Curran: Writing-review and editing; Ranjit S. Bindra: Writingreview and editing; Joseph N. Contessa: Writing-review and editing; Kirtesh R. Patel: Conceptualization, methodology, visualization, writing-original draft, supervision, project administration, and writing-review and editing.

\section{Acknowledgements}

The Authors would like to thank the American College of Surgeons Commission on Cancer and the American Cancer Society for access to the data that enabled this analysis.

\section{References}

1 Pouratian N and Schiff D: Management of low-grade glioma. Curr Neurol Neurosci Rep 10(3): 224-231, 2010. PMID: 20425038. DOI: $10.1007 / \mathrm{s} 11910-010-0105-7$

2 Bush NAO and Chang S: Treatment strategies for low-grade glioma in adults. J Oncol Pract 12(12): 1235-1241, 2016. PMID: 27943684. DOI: $10.1200 /$ jop.2016.018622

3 Buckner JC, Shaw EG, Pugh SL, Chakravarti A, Gilbert MR, Barger GR, Coons S, Ricci P, Bullard D, Brown PD, Stelzer K, Brachman D, Suh JH, Schultz CJ, Bahary J-P, Fisher BJ, Kim $\mathrm{H}$, Murtha AD, Bell EH, Won M, Mehta MP and Curran WJJ: Radiation plus procarbazine, CCNU, and vincristine in lowgrade glioma. N Engl J Med 374(14): 1344-1355, 2016. PMID: 27050206. DOI: 10.1056/NEJMoa1500925

4 Shaw EG, Berkey B, Coons SW, Brachman D, Buckner JC, Stelzer KJ, Barger GR, Brown PD, Gilbert MR and Mehta M: Initial report of radiation therapy oncology group (RTOG) 9802: Prospective studies in adult low-grade glioma (LGG). J Clin Oncol 24(18_suppl): 1500-1500, 2006. PMID: 27952315. DOI: 10.1200/jco.2006.24.18_suppl.1500

5 Karim AB, Maat B, Hatlevoll R, Menten J, Rutten EH, Thomas DG, Mascarenhas F, Horiot JC, Parvinen LM, van Reijn M, Jager JJ, Fabrini MG, van Alphen AM, Hamers HP, Gaspar L, Noordman E, Pierart M and van Glabbeke M: A randomized trial on dose-response in radiation therapy of low-grade cerebral glioma: European Organization for Research and Treatment of Cancer (EORTC) study 22844. Int J Radiat Oncol Biol Phys 36(3): 549-556, 1996. PMID: 8948338. DOI: 10.1016/S03603016(96)00352-5 
6 van den Bent MJ, Afra D, de Witte O, Ben Hassel M, Schraub S, Hoang-Xuan K, Malmstrom PO, Collette L, Pierart M, Mirimanoff $\mathrm{R}$ and Karim AB: Long-term efficacy of early versus delayed radiotherapy for low-grade astrocytoma and oligodendroglioma in adults: The EORTC 22845 randomised trial. Lancet 366(9490): 985-990, 2005. PMID: 16168780. DOI: 10.1016/s0140-6736(05)67070-5

7 Pignatti F, van den Bent M, Curran D, Debruyne C, Sylvester R, Therasse P, Afra D, Cornu P, Bolla M, Vecht C and Karim AB: Prognostic factors for survival in adult patients with cerebral low-grade glioma. J Clin Oncol 20(8): 2076-2084, 2002. PMID: 11956268. DOI: $10.1200 /$ jco.2002.08.121

8 International Agency for Research on Cancer: International Classification of Diseases for Oncology ICD-O-3 online. World Health Organization, 2019. Available at https://codes.iarc.fr/ codegroup/2. Last accessed on 7th May 2019.

9 Eyre HJ, Crowley JJ, Townsend JJ, Eltringham JR, Morantz RA, Schulman SF, Quagliana JM and al-Sarraf M: A randomized trial of radiotherapy versus radiotherapy plus CCNU for incompletely resected low-grade gliomas: A Southwest Oncology Group study. J Neurosurg 78(6): 909-914, 1993. PMID: 8487073. DOI: 10.3171/jns.1993.78.6.0909

10 Suneja G, Alonso-Basanta M, Lustig R, Lee JY and Bekelman JE: Postoperative radiation therapy for low-grade glioma: Patterns of care between 1998 and 2006. Cancer 118(15): 37353742, 2012. PMID: 22180333. DOI: 10.1002/cncr.26693

11 The Cancer Genome Atlas Research N: Comprehensive, integrative genomic analysis of diffuse lower-grade gliomas. N Engl Med 372(26): 2481-2498, 2015. PMID: 26061751. DOI: 10.1056/NEJMoa1402121
12 Schiff D, Brown PD and Giannini C: Outcome in adult lowgrade glioma: The impact of prognostic factors and treatment. Neurology 69(13): 1366-1373, 2007. PMID: 17893297. DOI: 10.1212/01.wnl.0000277271.47601.a1

13 Leu S, von Felten S, Frank S, Vassella E, Vajtai I, Taylor E, Schulz M, Hutter G, Hench J, Schucht P, Boulay J-L and Mariani L: IDH/MGMT-driven molecular classification of lowgrade glioma is a strong predictor for long-term survival. NeuroOncology 15(4): 469-479, 2013. PMID: 23408861. DOI: 10.1093/neuonc/nos317

14 Louis DN, Perry A, Reifenberger G, von Deimling A, FigarellaBranger D, Cavenee WK, Ohgaki H, Wiestler OD, Kleihues P and Ellison DW: The 2016 World Health Organization Classification of Tumors of the Central Nervous System: A summary. Acta Neuropathol 131(6): 803-820, 2016. PMID: 27157931. DOI: 10.1007/s00401-016-1545-1

15 Shaw EG, Berkey B, Coons SW, Bullard D, Brachman D, Buckner JC, Stelzer KJ, Barger GR, Brown PD, Gilbert MR and Mehta M: Recurrence following neurosurgeon-determined grosstotal resection of adult supratentorial low-grade glioma: Results of a prospective clinical trial. J Neurosurg 109(5): 835-841, 2008. PMID: 18976072. DOI: 10.3171/JNS/2008/109/11/0835

Received April 16, 2019

Revised May 7, 2019

Accepted May 8, 2019 